\title{
Indigeneidad en la política exterior de Bolivia en el gobierno de Evo Morales $(2006-2014)^{*}$
}

\author{
Amaya Querejazu Escobari*
}

Artículo recibido: 9 de octubre de 2014

Artículo aprobado: 5 de noviembre de 2014

Doi: dx.doi.org/10.12804/desafios27.01.2015.05

Para citar este artículo: Querejazu E., A. (2015). Indigeneidad en la política exterior de Bolivia en el gobierno de Evo Morales (2006-2014). Desafíos 27(1), 159-184. doi: dx.doi. org/10.12804/desafios27.01.2015.05

\section{Resumen}

En este artículo se analiza la inclusión de la indigeneidad en la politica exterior de Bolivia, en el gobierno de Evo Morales. Se plantea que la indigeneidad se utiliza en la política exterior, primero, para justificar una actitud revisionista e incluso subversiva, respecto a la posición del país en temas de la agenda internacional y justificar asi una alternativa al modelo neoliberal capitalista desde las cosmovisiones indigenas, siendo un ejemplo el de suma qamaña. Segundo, la indigeneidad se usa como un factor constitutivo de identidad, dirigido no solo al plano internacional, sino también a la misma población boliviana. En la primera parte se hace una breve contextualización de los cambios en el país desde la llegada al gobierno de Morales en el año 2006. Luego se describe la política exterior en el pasado y los puntos de quiebre que se ban dado en el último gobierno, esto para determinar si la presencia

\footnotetext{
* Una versión preliminar de este artículo fue presentada en el Tercer Congreso de la Red Colombiana de Relaciones Internacionales (Redintercol), realizado en la Universidad Eafit de Medellín, el 27 y 28 de febrero de 2014.

** Profesora de la Facultad de Derecho y Ciencias Políticas de la Universidad de Antioquia. Estudiante de doctorado en Ciencia Política en la Universidad de los Andes. Correo electrónico: amaya.querejazu@gmail.com
} 
de la indigeneidad en la politica exterior del país es un resultado de la identidad de la nación o una estrategia pragmática del gobierno. Finalmente, se analizará la política exterior indígena e indigenista de Bolivia. Se concluye que la política exterior del gobierno de Morales está llena de contradicciones y que se caracteriza por una tensión entre el revisionismo y el pragmatismo.

Palabras clave: Bolivia, política exterior, indigeneidad, suma qamaña, interés nacional.

\title{
"Indigenidad" in Bolivia's Foreign Policy During Evo Morales' Government
}

\begin{abstract}
This paper analyzes the inclusion of "indigenidad" in Bolivia's foreign policy during the government of Evo Morales. It argues that indigenidad is used at least for two purposes: first, to justify a rather revisionist-even subversive-position of the country regarding issues of the international agenda in order to propose an alternative to the neoliberal model from the perspective of indigenous worldviews, such as "suma qamaña". Second, indigenidad is used as a constitutive factor for identity that, in turn, is not necessarily conceived for the international level, but for domestic andiences. The first part of the article contextualizes the process of change and transformation of the country since Morales became president in 2006, in order to assess the purpose of the use of indigenidad. The second part analyzes Bolivia's "indigenous" foreign policy. The overall conclusion is that Bolivia's foreign policy is the result of a tension between revisionism and pragmatism.
\end{abstract}

Keywords: Bolivia, foreign policy, indigenidad, suma qamaña, national interest.

\section{Indigeneidade na política exterior da Bolívia no governo de Evo Morales (2006-2014)}

\section{Resumo}

Este artigo analisa a inclusão da indigeneidade na política exterior da Bolívia no governo de Evo Morales. Expõe-se que a indigeneidade utiliza-se na política exterior, primeiro, para justificar uma atitude revisionista e inclusive subversiva, respeito à posição do país em temas da agenda internacional e justificar assim uma alternativa 
ao modelo neoliberal capitalista desde as cosmovisões indígenas, sendo um exemplo o Suma Qamaña. Segundo, a indigeneidade se usa como um fator constitutivo de identidade, dirigido não só ao plano internacional, mas também a mesma população boliviana. Na primeira parte se faz uma breve contextualização das mudanças no país desde a chegada ao governo de Morales en 2006. Depois descreve-se a politica exterior no passado e os pontos de ruptura que se têm dado no último governo; isto, para determinar se a presença da indigeneidade na politica exterior do pais é um resultado da identidade da nação ou uma estratégia pragmática do governo. Finalmente, se analisará a política exterior indígena e indigenista da Bolivia. Conclui-se que a política exterior do governo de Morales está cheia de contradições e que caracteriza-se por uma tensão entre o revisionismo e o pragmatismo.

Palavras-chave: Bolivia, politica exterior, indigeneidade, suma qamaña, interesse nacional.

\section{Introducción}

La indigeneidad es un concepto ampliamente tratado en la antropología, pero prácticamente ausente en la disciplina de las Relaciones Internacionales (Shaw, 2002) y aún más en el análisis de política exterior. Puede afirmarse que es un concepto contingente y aún bastante discutido. Según Merlan, la indigeneidad connota la pertenencia, lo originario y los procesos profundamente sentidos de apego e identificación. La definición de indigeneidad tiene dos categorías, por un lado, una relacional, donde el énfasis está en la relación entre los indígenas y los otros; y otra de criterio, que hace referencia a las propiedades inherentes a quienes se llaman indígenas, a las condiciones que permiten una identificación de lo indígena como una especie global (global kind) y, por lo tanto, con una dimensión internacionalizada. Estas dos categorías no son fácilmente distinguibles y, en algunos casos, una predomina sobre la otra (Merlan, 2009). También puede entenderse la indigeneidad como un término creado de frente a la conquista, aspecto que todos los pueblos indígenas del mundo tienen en común, pero que al mismo tiempo marca las diferencias entre ellos de acuerdo sus experiencias de colonialismo (Vecchione, 2009). La indigeneidad es vivida de diferentes maneras en el mundo, por lo que no es una categoría unitaria, y admite que muchas ver- 
siones de identidad indígena puedan coexistir, de ahí que adquiere significado, no de propiedades esenciales, sino en relación con lo que no se considera indígena (De la Cadena \& Stran, 2007). Siendo un concepto tan amplio, abarca el estudio de los pueblos indígenas, nativos u originarios, el indigenismo (Trigger \& Dalley, 2010) y la indianidad, que es la utilización estratégica y con fines pragmáticos de la identidad étnica y de una noción que se usó para someter, para lograr el reconocimiento de ciertos derechos (Laurent, 2005) o lograr el reconocimiento de los pueblos indígenas como actores sociales y políticos a partir de mostrarse a sí mismos como más primitivos y exóticos (Franke, 2009). Para el caso específico de los Andes, De la Cadena define la indigeneidad como un proceso de formación compleja, una articulación histórico-política de más de uno, menos de dos, mundos socionaturales. Como formación histórica, la indigeneidad andina no desapareció ni con el cristianismo primero, ni con la ciudadanía (mestizaje), aunque tampoco fue impermeable a ellos. Por lo tanto, desde ese punto de vista la indigeneidad siempre ha sido parte de la modernidad y, al mismo tiempo, diferente y nunca modernista (De la Cadena, 2010).

Incluir la indigeneidad en una política exterior es, sin duda, algo novedoso e interesante, por lo que este artículo busca analizar este fenómeno en el caso de Bolivia y el gobierno de Evo Morales. Se argumenta que no solo se trata de la introducción de un tema no tradicional en la agenda de política exterior, sino que además se presenta como un interés nacional que busca reivindicaciones históricas. Esto es utilizado por el gobierno para legitimarse como líder en ciertos temas, como los relacionados con el medio ambiente; diversificar su política exterior y llegar a otros pueblos indígenas y grupos ambientalistas de otros países. En segundo lugar, el factor de indigeneidad es un elemento utilizado en la constitución de identidad con el objeto de construir nación. A partir de eso, un pilar fundamental de la política exterior está orientado a proponer alternativas al modo de vida occidental y al modelo económico neoliberal, usando un sistema ético de valores de los pueblos y sus cosmovisiones en lo que se conoce como suma qamaña, vivir bien o buen vivir. Si bien, esto no está teniendo eco en la relación con otros estados, sí ha despertado el interés de muchos 
grupos sociales, académicos y activistas que ven en esta propuesta una oportunidad para que por fin sea un estado el que lleve esa agenda a nivel internacional, lo que abre la posibilidad de cuestionar el orden internacional más allá de lo político ideológico y de la economía internacional e imaginar lo internacional de otra forma.

Claramente, la presencia de la indigeneidad en la política exterior de un país no solo es algo llamativo y novedoso, sino también algo sumamente complejo. A nivel doméstico, la utilización de la indigeneidad, como un imaginario colectivo, factor de cohesión de la sociedad, lleva a que la cosmovisión de unos pueblos se imponga sobre otros y se reproduzcan los procesos de colonialidad. Además, recurrir a la indigeneidad ha causado fisiones sociales que van más allá de las tradicionales divisiones ideológicas y, a la larga, más que un factor de cohesión social, puede ser un factor de rupturas. En un país como Bolivia, y teniendo en cuenta que los conceptos relacionados con la indigeneidad son susceptibles de cambio y de diferentes interpretaciones, existen muchas formas de entender lo indígena.

Con respecto a la política exterior, las contradicciones no se hacen esperar en un discurso indigenista que muestra, por un lado, al estado y sus propuestas internacionales como defensores del medio ambiente y de la vida en armonía con los recursos y los pueblos y, por otro, la política económica, comercial y de desarrollo que atenta contra los intereses y valores de los pueblos que dice representar. Estas contradicciones están dadas por la fricción y la división de los actores domésticos, pero también son propias de las cosmovisiones indígenas, son parte de un todo. Este es, sin duda, otro desafío para pensar la política exterior. Esta complejidad lleva a pensar que la utilización de la indigeneidad en la política es un arma de doble filo, con consecuencias tanto a nivel doméstico, como internacional.

Vale la pena aclarar que no hay mucha documentación sobre este fenómeno, desde el punto de vista del análisis de política exterior. A su vez, puede decirse que los marcos conceptuales tradicionales de análisis de política exterior no son del todo útiles para analizar un factor como el de la indigeneidad en este tema, por lo que se acudirá 
a aquellos enfoques que estudian y analizan los factores de identidad y de ideas en la política exterior, en la medida que la indigeneidad es utilizada para la construcción de una identidad nacional.

El trabajo está estructurado de la siguiente manera. En primer lugar, se hará una breve contextualización del proceso de cambio y transformación del país desde la llegada al gobierno de Evo Morales en el 2006, se mencionarán algunas reformas institucionales importantes para la articulación de la política exterior. La importancia de esto radica en que los factores domésticos de cambio sirven para contextualizar y ayudan a entender por qué y cómo se utiliza la indigeneidad en la política exterior, sobre todo en su dimensión internacional. La indigeneidad es usada, tanto a nivel doméstico como internacional, como una forma de recuperar memoria, iniciar o continuar con procesos de descolonización, aspectos que tienen origen tanto doméstico como internacional. En segundo lugar, se hará una descripción de la política exterior en el pasado y los puntos de quiebre que se han dado en el último Gobierno, y se ilustrará el uso de la indigeneidad en la política exterior para determinar si éste es un aspecto que realmente refleja la identidad de la mayoría de la población del país o una estrategia del gobierno. Esto permitirá analizar si la presencia de la indigeneidad en la política exterior del país es un interés nacional, resultado de la identidad del país, o si es resultado de una estrategia de Gobierno. Finalmente, se analizará la política exterior indígena e indigenista de Bolivia, su posible alcance y los obstáculos y complejidades que tiene.

\section{Bolivia a partir de 2006 ¿cambio o transformación?}

La llegada al poder del primer presidente indígena en un país tan diverso como Bolivia posibilitó, en cierta medida, la concreción de muchos procesos de profunda transformación política y social, largamente postergados, que habían comenzado a gestarse desde algunos años atrás (Patzi, 2009) y que terminaron por plasmarse en la Constitución Política de 2009. Se trata pues de un hito importante que simboliza la refundación del país, se sustituye la República por el Estado Plurinacional, lo que apunta a ir más allá del reconocimiento de la pluralidad étnica y cultural, y recuperar la memoria ancestral de 
los pueblos, acallada durante quinientos años de colonia y doscientos de República. Un ejemplo de esto es el primer párrafo del preámbulo de la constitución:

En tiempos inmemoriales se erigieron montañas, se desplazaron ríos, se formaron lagos. Nuestra amazonia, nuestro chaco, nuestro altiplano y nuestros llanos y valles se cubrieron de verdores y flores. Poblamos esta sagrada Madre Tierra con rostros diferentes, y comprendimos desde entonces la pluralidad vigente de todas las cosas y nuestra diversidad como seres y culturas. Así conformamos nuestros pueblos, y jamás comprendimos el racismo hasta que lo sufrimos desde los funestos tiempos de la colonia (Nueva Constitución Política del Estado Boliviano, 2009).

Este párrafo ilustra el argumento de Vecchione (2009), donde afirma que la indigeneidad pasa por la experiencia colonial. También simboliza la reivindicación de aspectos propios del modo de pensar indígena y se hace alusión a la reconexión de lo social y político con lo natural, o espacios de interconexión parcial (De la Cadena, 2010), donde los seres no-humanos asumen la vida que tienen de acuerdo con las cosmovisiones indígenas.

A partir de esto, se dieron también una serie de reformas institucionales y legales que se orientan a revalorizar lo indígena en el país. Esta transformación desde arriba pasa por la reforma educativa, que ha consistido en la recuperación de los conocimientos científicos ancestrales, en recontar la historia, al reivindicar el legado indígena, con un objetivo revisionista, lo que apunta a un proceso de descolonización de los saberes sin negar el legado occidental. ${ }^{1}$ El Ministerio de Educación se apoya en el trabajo de tres viceministerios que son estratégicos en este proceso de transformación: el Viceministerio de Ciencia y Tecnología, que está a cargo, entre otras tareas, de la recopilación de los saberes ancestrales y su reivindicación como ciencia;

\footnotetext{
1 Por ejemplo, si antes los himnos hacían referencia a los próceres de la independencia, hoy el empleo de himnos es en homenaje a líderes y sublevaciones indígenas y deben enseñarse obligatoriamente junto con el himno de la república.
} 
el Viceministerio de Educación Alternativa, y el Viceministerio de Educación regular, estos dos últimos tienen como misión la educación plural y descolonizadora.

Por otra parte, se creó el Viceministerio de la Descolonización, con la misión de visibilizar procesos históricos olvidados y ubicarlos en un plano de igualdad frente a la versión occidental de la historia boliviana. Otras reformas institucionales importantes son la creación del Viceministerio de la Coca y de Desarrollo Integral, que tiene como fin potencializar y divulgar el valor ancestral, cultural y medicinal de la hoja de coca (y de otros cultivos ancestrales) y apoyar la industrialización de sus usos. Esto tiene la finalidad clara de desmitificar su estigmatización occidental como narcótico ilegal y de asociarla con procesos de desarrollo económico, social y cultural del país.

La actividad de esos viceministerios se concatena con la política exterior, que también, a partir de la utilización de símbolos, elementos indígenas y conocimientos ancestrales, ha formulado una política exterior de la que hacen parte como pilares fundamentales la diplomacia de los pueblos y la comunidad para vivir bien (suma qamaña).

Todo esto muestra el carácter revisionista del gobierno de Evo Morales, con el ánimo de recuperar la memoria histórica del país y la estima de los pueblos indígenas. En el ámbito internacional, esta actitud revisionista ha consistido en replantear los compromisos y la posición del país, asumidos por gobiernos anteriores. Se entiende, entonces, que es un proceso tanto doméstico como internacional, que pasa por la construcción de nación y de identidad. Un proceso que ha sido no solo liderado por el gobierno, sino diseñado por él. Esto quiere decir que se ha instrumentalizado la indigeneidad de muchas maneras. Para empezar, puede decirse que los quechua y aymara - grupos mayoritarios y políticamente más activos y con más experiencia en la movilización social— tienen, pese a sus múltiples divergencias internas propias, un mayor impacto e influencia en el gobierno. De esta manera, la pluralidad indígena que tanto se menciona en los discursos oficiales termina siendo aymarocentrista o andinocentrista. Al ser un proyecto que se implementa desde arriba, 
el Gobierno utiliza sus propias interpretaciones de lo indígena, al excluir la participación de amplios sectores sociales, entre ellos, los indígenas que no comparten la visión o interpretación de quienes diseñan esos métodos de descolonización.

Sin embargo, vale la pena resaltar que la utilización de la indigeneidad ha significado, también, una ventana de oportunidad para algunos actores. Las instituciones antes mencionadas son, a su vez, utilizadas por algunos grupos y pueblos para llamar la atención y obtener recursos, por ejemplo, se han dado casos en los que los grupos han inventado leyendas, conocimientos y hasta idiomas para lograr acceder al reconocimiento y los derechos que esto conlleva. ${ }^{2}$ Es evidente que la revalorización de lo indígena viene acompañada de la indianidad, que, como se dijo, es una utilización estratégica de lo indígena para lograr acceder a espacios políticos y recursos económicos de indigenización de fenómenos de la cultura popular, hibridados por el paso del tiempo, y de la reinvención o resemantización de lo indígena.

No es de extrañar, entonces, que los conocedores de las culturas y creencias ancestrales acusen la tergiversación de estas con fines políticos por parte del gobierno. Según Huarachi Yampara (2011), por ejemplo, Bolivia no está atravesando por una transformación (pachakuti), sino solo por un cambio, un cambio de guardia de gobierno de derecha a uno de izquierda, aunque remozado de indigenismo. Es un gobierno prisionero de las ideas de izquierda y no un gobierno indígena, como generalmente se piensa. Por su parte, Felipe Quispe, reconocido líder indígena y campesino, se opone abiertamente a esta utilización de la indigeneidad y ha hecho manifiesta su molestia frente al Gobierno de Morales. Para Quispe, que se considera katarista ${ }^{3}$ indianista, no puede considerarse que Morales haya beneficiado a los indígenas ni que sea pro-indígena (Quispe, 2014).

\footnotetext{
2 Rodrigo Hoz de Vila, Jefe de Ciencia y Tecnología del Viceministerio de Ciencia y Tecnología de Bolivia. Octubre 2013.

3 Según Quispe, que es abiertamente uno de los líderes indígenas más radicales, el movimiento indio siempre ha tenido dos brazos: un brazo democrático y legal, con el que se participa en las elecciones y otro que sigue el camino de Túpac Katari y Túpac Amaru, de lucha armada.
} 
Esta posición permite ilustrar cómo la indigeneidad es objeto de politización entre los distintos actores sociales del país, entre indigenistas que apelan a la indianidad y otros que piensan en la indigeneidad como algo más espiritual. Yampara reconoce que en lo único que hay un cambio es en la autoestima del indígena, pero critica que la idea de lo plural es algo que esta únicamente en el discurso. Si bien, los resultados de estos cambios aún están por verse (Kohl, 2010), es evidente que la creciente consciencia indígena y la autoestima indígena han marcado un cambio que no puede sino entenderse como un acto de justicia conquistada a pulso (González, 2007).

Esto demuestra por qué la indigeneidad es un concepto tan elusivo. Necesariamente, la indigeneidad también tendrá que ser negociada a partir de las distintas interpretaciones si no se quiere que sea una creación del Gobierno que, además, se queda solo en el discurso. Sin embargo, muestra la importancia que tiene el nivel doméstico para interpretar y entender una política exterior y la dimensión internacional de la indigeneidad que se analizarán más adelante. Lo doméstico es tanto fuente como destinatario del discurso indigenista y de la utilización de la indigeneidad. A continuación, se hace referencia a los cambios que ha sufrido la política exterior boliviana, tanto en temas tradicionales de la agenda, como por consecuencia de la utilización de la indigeneidad.

\section{Política exterior boliviana}

Históricamente, la política exterior boliviana se ha caracterizado por su cercanía a los intereses de Estados Unidos, con algunos periodos de tensión o distanciamiento. El Gobierno de Evo Morales muestra una ruptura con este patrón. Los ejemplos más evidentes son la expulsión del embajador norteamericano en $2008^{4}$ y el cambio en la política antidrogas (la agencia antidrogas DEA fue cerrada definitivamente el 2008); el importante paso de la erradicación a la interdicción de la

\footnotetext{
4 Hasta el día de hoy no existen embajadores de ninguno de los países en las respectivas legaciones. En el 2013, luego del episodio del "secuestro" del avión presidencial en el que viajaba Evo Morales, la tensión entre ambos países se agudizó y Morales amenazó con el cierre de la embajada.
} 
hoja de coca; el desarrollo alternativo integral y la no represión a los campesinos; así como el rechazo a la posibilidad de establecer bases militares estadounidenses en el territorio nacional. El aspecto subversivo de la política exterior, no solo tiene un discurso y una posición anti neoliberal y antimperialista frente a los Estados Unidos - que es muy usual en la región-, sino que la expulsión de la DEA y el cierre de USAID se suman a la aceptación por parte del Gobierno boliviano de la no renovación de las preferencias arancelarias en el marco del APTDEA. La contestación no se quedó en el discurso y, pese a los importantes costos económicos y políticos que significaron estas acciones para Bolivia, ni la zanahoria ni el garrote fueron instrumentos idóneos para direccionar al país hacia los intereses de Estado Unidos, lo que puede, sin duda, leerse como un triunfo: "Evo morales se ha convertido en un ejemplo de la rebeldía y de la soberanía nacional con las cuales la inmensa mayoría de la población, aun opositora al gobierno, se siente identificada" (Moldiz, 2007, p. 174).

Por otra parte, la actitud revisionista del Gobierno de Morales ha replanteado, incluso, la única política de Estado (Escobari, 1999) que tenía el país respecto a la reivindicación marítima frente a Chile. Asumiendo una actitud diferente, y luego de décadas de intentos fallidos de negociación bilateral y mediación multilateral, así como la suma de otros episodios que agravaron la relación bilateral, el gobierno boliviano decidió cambiar de estrategia, al dejar de lado los intentos de negociación y optar por el arreglo judicial como la instancia adecuada para resolver la disputa. De esta manera, solicitó en una demanda ante la Corte Internacional de Justicia de la Haya en 2013, la restitución del territorio perdido como consecuencia de una guerra. Esta decisión contó con un apoyo incuestionable de todos los sectores y regiones del país.

La política de cambios de Morales ha sido polémica también por otras razones, entre las más importantes, el cambio de modelo ideológico, político y económico imperantes hasta el momento. La terminación de las preferencias arancelarias del APTDEA ha creado la necesidad del sector productivo de abrirse nuevos mercados, construir relaciones comerciales con nuevos socios y fortalecer las que tenía con otros 
países. Con Evo Morales, Bolivia se ubica dentro del grupo de países latinoamericanos que han optado por un nuevo giro a la izquierda como Venezuela, Brasil, Argentina, Ecuador y Nicaragua (Rochlin, 2007; Maira, 2007).

Puede decirse entonces que la política exterior de Morales va en contra de los intereses de la élite política que había gobernado por décadas y había construido el tipo de relaciones que hoy se cuestionan. Sin embargo, las contradicciones no se hacen esperar, los grandes cambios en la política extractiva, de los recursos naturales que Bolivia exporta, entre ellos gas, estaño y litio, han sufrido un cambio de régimen a nivel interno como consecuencia de las políticas de nacionalización. Sin embargo, a nivel internacional Bolivia sigue manteniendo un papel de exportador de recursos primarios y los contratos con inversionistas multinacionales aún forman parte del esquema. Asimismo, se ha apostado a formar parte de la agenda de desarrollo económico social de la Alianza Bolivariana para los Pueblos de Nuestra América del - Tratado de Comercio de los Pueblos, con importantes costos y desventajas para el incipiente sector productivo del país (Rochlin, 2007), que en gran medida dependía de las preferencias otorgadas por Estados Unidos. El objetivo central de la presidencia de Morales ha sido romper con el pasado y moverse hacia el futuro sin una hoja de ruta clara, con una atención primaria en los asuntos domésticos, más que a la política exterior (Birns \& Sánchez, 2011). Las relaciones económicas y comerciales de Bolivia tienen un ingrediente ideológico no tan radical como las de Venezuela, y la decisión de mantener la inversión extranjera en los recursos energéticos ha sido sin duda pragmática. Como resultado, Birns \& Sánchez (2011) afirman que Morales no ha sido muy arriesgado en sus iniciativas de política exterior, que es más pragmática que ideologizada.

Se evidencia entonces que los planteamientos subversivos buscan una ruptura a partir de revisar y cuestionar las relaciones pasadas del país; así como el interés de mantener —en gran medida por necesidad-las relaciones comerciales tradicionales. La tensión dependencia/independencia es clara: el Gobierno necesita de los recursos de las regalías para financiar su proyecto de independencia. Así es como Bolivia 
muestra que la contradicción no necesariamente es problemática y que una política exterior puede ser subversiva y pragmática a la vez. Es más, este tipo de contradicciones en el ámbito económico y político no son nuevas en la región, que históricamente se ha debatido entre una búsqueda de autonomía e independencia de Estados Unidos.

Lo que sí es nuevo es la inclusión de la indigeneidad que, además de considerar el tema indígena como parte de la agenda internacional, busca visibilizar diferentes sistemas de valores, conocimientos y cosmovisiones ancestrales largamente callados por la colonialidad y utilizarlos, tanto para abrirse espacios y lograr cierto protagonismo, como para proponer una alternativa al modelo neoliberal occidental. Pero las formas y mecanismos usados, en cierta medida, van más allá de una utilización nominal de la indigeneidad y sí que incluyen aspectos propios de las cosmovisiones. Un ejemplo clave de esto es el Proyecto de la Carta sobre los Derechos de la Madre Tierra que Bolivia ha llevado a las Naciones Unidas para la ratificación de otros estados, pues no solo se trata de asumir la iniciativa, sino de realmente intentar dar subjetividad política y jurídica a otros mundos, no solo las cosmovisiones de los pueblos, sino el mundo animal, el mundo vegetal y el mundo de las espiritualidades (De La Cadena, 2010). Se trata a la tierra como titular de derechos, aspecto que en occidente es concebible solo para los ciudadanos (humanos).

De todas formas, si bien es poco probable que esta convención vaya a cambiar el enfoque internacional hacia el cambio climático, le ha permitido a Bolivia ponerse a la cabeza como representante de las demandas indígenas y ambientales del Sur Global, aumentando su perfil internacional (Birns \& Sánchez, 2011). Es decir, que si los estados no reconocen la posibilidad de que la tierra sea titular de derechos, por otro lado, sí existe una conciencia internacional, reivindicada por los pueblos indígenas en general, de que la tierra sí debería considerarse sujeto de derechos y que ellos son sus custodios (Doolittle, 2010). Si un estado como Bolivia asume esa vocería, puede lograr llegar a contar con el apoyo en negociaciones ambientales de los pueblos y grupos ambientalistas que no tienen voto en las toma de decisiones. Esto muestra, además, la dimensión internacional de la indigeneidad. 
Otro ejemplo, que sirve para mostrar la presencia de la indigeneidad en la política exterior revisionista de Bolivia, se da frente a la Convención Única sobre Estupefacientes de 1961. Bolivia denunció el tratado para posteriormente adherirse con reservas en lo que se refiere a la prohibición de la práctica de masticar la hoja de coca. $\mathrm{Al}$ respecto, uno de los objetivos estratégicos del gobierno en materia de política exterior es "lograr que la comunidad internacional reconozca a la hoja de coca originaria y ancestral como patrimonio cultural y recurso natural renovable de la biodiversidad de Bolivia y como factor de cohesión" (Choquehuanca, 2012). El derecho ancestral a masticar la hoja de coca forma parte de los intereses nacionales, por ser parte de la historia y religiosidad.

Por otra parte, el hecho de que la Organización de Naciones Unidas (ONU) y la organización de Naciones Unidas para la Alimentación y la Agricultura (FAO) declararan 2013 como el Año Internacional de la Quinua significó para Bolivia una nueva oportunidad para introducir la indigeneidad en lo que a primera vista parece un tema puramente comercial o de alimentación. A partir de este hecho, Bolivia, bajo el liderazgo del Ministerio de Relaciones Exteriores, ha iniciado un programa llamado "Un futuro sembrado hace miles de años". $5 \mathrm{Al}$ margen de que Bolivia es uno de los principales exportadores del valioso grano, cada vez más apetecido y conocido en el resto del mundo, este tiene un valor simbólico importante en las cosmovisiones andinas que lo consideran un grano sagrado, un "grano de oro". Su utilización es frecuente en rituales y tiene fines medicinales. Con el lema y con las diferentes estrategias que se desarrollan en el programa, se busca reivindicar ambas dimensiones de la quinua, la comercial y la simbólica.

Asimismo, es evidente la combinación de aspectos ideológicos, como el giro a la izquierda enmarcado en el Socialismo del Siglo XXI, marcadamente anti neoliberal, con factores propios de las cosmovisiones

\footnotetext{
5 El programa incluye congresos, simposios, recorridos por rutas de la quinua y demás actividades. La información sobre el programa se encuentra disponible en la página: http:/ / www.quinuainternacional.org.bo/
} 
indígenas, aspecto que se plasma en una propuesta de cambio en la forma de vida. A partir de la reivindicación de conocimientos y valores ancestrales indígenas, se cuestiona el consumismo y, en general, los valores occidentales que propugnan por el progreso humano y el éxito en una constante búsqueda de vivir mejor y estar mejor. Según Huanacuni, la humanidad está atravesando por una crisis de vida, donde impera lo desechable, seres solos, individualistas, hijos sin padres, consumismo infrenable. "Una cultura del respeto y de la vida es una respuesta que ha sido guardada por los pueblos y hoy está siendo compartida al mundo como un nuevo paradigma de vida, porque en la forma de vida que propone Occidente no hay futuro" (Huanacuni, 2012).

De esta manera, bajo la propuesta llamada suma qamaña o vivir bien, el gobierno se apropia de estas formas alternativas de pensar e inserta en su política exterior valores ancestrales indígenas para cumplir un papel antisistémico, al mostrar alternativas al neoliberalismo, al capitalismo y al neoimperialismo. suma qamaña consiste en plantear otros principios de coexistencia y de convivencia, como llegar a acuerdos en consenso, respetar las diferencias, vivir en complementariedad, equilibrio con la naturaleza, defender la identidad, aceptar las diferencias, priorizar derechos cósmicos, saber comer, saber beber, saber danzar y saber trabajar, retomar el abya yala, ${ }^{6}$ saber comunicarse, ejercer control social, trabajar en reciprocidad, no robar y no mentir, proteger las semillas, respetar a la mujer, vivir bien y no mejor, recuperar recursos, ejercer la soberanía, aprovechar el agua, escuchar a los mayores (Choquehuanca 2012; Huanacuni, 2012).

\section{Suma qamaña, o}

[...] el concepto del "Vivir Bien" andino solo puede ser comprendido a cabalidad como expresión de una cosmovisión y filosofía distintas a la occidental dominante, y no como receta

\footnotetext{
6 El término Abya Yala tiene varios significados, entre ellos "tierra nueva" o "tierra madura". Pertenece a la lengua kuna, pero ha sido retomado por los pueblos y movimientos indígenas del continente para designar al continente antes de la llegada de los europeos.
} 
económica, ecológica o cultural. En este sentido, se trata de una metáfora que representa otro modelo civilizatorio que se presenta como alternativa ante la decadencia y el agotamiento del modelo dominante de la modernidad occidental y el ideal capitalista que esta conlleva (Esterman 2012, p. 11).

En una especie de suma qamaña for export, se reivindica un "queremos volver a ser", proponiendo cambios en el tiempo hacia un equilibrio y armonía, no solo entre los pueblos, sino entre el hombre y la naturaleza (Choquehuanca, 2012). Ahora bien, no es muy clara la forma en la que el Gobierno plantea esto como una verdadera alternativa al modelo neoliberal o si tiene una estrategia clara para hacerlo. Por ahora, la relación entre estos valores y la posición antisistémica frente al capitalismo neoliberal es una tarea pendiente, pues el gobierno no ha establecido un plan de acción concreto o actividades para implementar esta propuesta, quedando por ahora en el discurso oficial.

Ahora bien, el papel de las embajadas en ese sentido ha variado a partir de fomentar actividades como la promoción del año nuevo andino, las universidades indígenas, la organización de conferencias de expertos sobre cosmovisión andina, entre otras cosas. En principio, actividades como estas se enmarcarían en la agenda cultural de cualquier embajada, pero la presencia de lo indígena sin duda busca ir más allá de lo folclórico y cultural. ${ }^{7}$ Lo cierto es que estas actividades son atractivas para muchas personas y son bien recibidas por grupos sociales que también cuestionan el modo de vida occidental neoliberal, lo que muestra una forma diferente de acercarse a nuevas audiencias en diferentes países a partir de la utilización de la indigeneidad.

Así como se planteó al principio, dicho uso de la indigeneidad es un arma de doble filo, de ahí la necesidad de hacer referencia a que,

\footnotetext{
7 Por ejemplo, es por medio del trabajo de las embajadas que se está dando a conocer un cambio en el calendario, el año nuevo ya no es únicamente el 31 de diciembre como es la tradición occidental, sino que ahora se celebra también el 21 de junio, día del solsticio de verano e inicio del año según la cosmovisión andina. Véase por ejemplo http://www. embajadaboliviacolombia.org/2014/02/24/ceremonia-de-permiso-a-la-madre-tierra-enfacatativa/
} 
si bien a nivel internacional Bolivia asume una posición no solo de defensora de los derechos de los indígenas sino también de vocería, a nivel doméstico la historia es muy distinta. Mientras que la política en defensa de los derechos de la Madre Tierra se vende como una política medioambiental, la agenda de desarrollo e infraestructura ha llevado al gobierno a poner el tema ambiental en un segundo plano, con consecuencias nefastas para algunas comunidades indígenas. Basta mencionar el problema del TIPNIS, un conflicto que aún no termina y que comenzó con la decisión del gobierno de construir una carretera que atraviesa el Parque Nacional Isiboro Sécure, lo que afectaría la vida de los pueblos indígenas que ahí habitan. Esta situación, que da cuenta de la doble moral del Gobierno a nivel doméstico e internacional, ha detonado importantes movilizaciones de oposición y de acusación frente a estas prácticas que cuentan con la participación de amplios sectores de la población civil y que a su vez demuestran un profundo malestar de las poblaciones indígenas. Por otro lado, mientras que Bolivia se muestra como un país promotor de los derechos de los pueblos indígenas, la misión de Naciones Unidas en el marco del el Foro Permanente para las Cuestiones Indígenas, que tuvo lugar en abril y mayo de 2009, constató:

[...] una vez más la existencia de servidumbre, trabajo forzoso y abusos contra pueblos indígenas, particularmente miembros del pueblo Guaraní, en las haciendas del Chaco y un empeoramiento muy significativo de las condiciones de vida del pueblo Guaraní debido a los ataques y amenazas a líderes, expulsiones a familias de líderes interpelando al gobierno boliviano para acabar con las violaciones a los derechos de los pueblos indígenas en el Chaco. ${ }^{8}$

Estos son solo algunos ejemplos concretos de lo que en general ha despertado reacciones de rechazo e indignación ante un uso utilitarista e hipócrita de lo que para muchos es aún un aspecto pendiente

\footnotetext{
8 Foro Permanente de las Naciones Unidas para las Cuestiones Indígenas. Misión a Bolivia. Informe y recomendaciones. Abril- mayo de 2009 Disponible en: http://www.un.org/esa/ socdev/unpfii/documents/UNPFI_Mission_Report_Bolivia_ES.pdf
} 
de reivindicación y reconocimiento. ¿Dónde quedan entonces los valores que se pretenden defender con la comunidad para vivir bien?

\section{La indigeneidad como factor constitutivo de identidad e interés nacional}

Como se ilustró a lo largo de este trabajo, la indigeneidad es utilizada de muchas maneras, entre ellas, como una forma de construir identidad e interés nacional, no solo frente a la población nacional, sino también respecto al papel que el Estado asume en el ámbito internacional. Vale la pena aclarar, sin embargo, que ambos conceptos -identidad e interés nacional- son también elusivos y complejos, sobre todo al momento de analizar la política exterior. A su vez, el peso que en el análisis y estudio de la política internacional y de la política exterior tiene el interés nacional depende necesariamente del enfoque teórico que se adopte. En el caso del interés nacional, para el realismo clásico, el interés nacional es un aspecto fundamental (Morgenthau, 1990), mientras que para otros casos, como el marxismo, es solo un medio para racionalizar e incluso enmascarar decisiones que se toman por muchas otras razones (Burchill, 2005). En su forma más simple, el interés nacional son las necesidades y deseos de un estado soberano en relación con otros estados comprendidos en el entorno internacional (Neuchterlein, 1976). Puede decirse que es por medio del concepto del interés nacional que los tomadores de decisión entienden las metas que deben perseguirse en la política exterior de un estado, siendo en la práctica la base de toda su acción. Pero, además, funciona como una herramienta teórica mediante la que se genera legitimidad y apoyo político. Por eso es que el interés nacional tiene un poder considerable, pues ayuda a constituir y legitimar las acciones de los estados (Weldes, 1996).

En cuanto a la identidad, en algunos trabajos relativamente recientes sobre análisis de política exterior como el de Dunne, Hadfield y Smith (2011) y Alden y Aran (2012), la identidad cumple un papel muy secundario, como un factor que se menciona y no una categoría de análisis. Aspectos como los conflictos de identidad - que serían bastante ilustrativos, para el caso boliviano-, cambios de identidad 
y relaciones de grupo basados en otras disciplinas todavía deben articularse al análisis de política exterior y ser más investigados (Kaarbo, 2003).

Sin embargo, pese a que no ha sido lo suficientemente analizada en política exterior, la importancia de la identidad en la política internacional es ampliamente reconocida. Entre los enfoques predominantes racionalistas y constructivistas ha habido discusiones importantes al respecto. La diferencia está en que los primeros la asumen como una variable independiente o dada, que explica otros aspectos de las relaciones internacionales, más que una variable a ser explicada. Estos enfoques despliegan la identidad estratégicamente como un recurso como cualquier otro, simplemente para satisfacer sus intereses (Katzenstein, 1996). Por su parte, los constructivistas, al asumir que la realidad es una construcción social donde prima la intersubjetividad, se interesan por la naturaleza de la identidad e intentan rastrear los procesos de constitución y cambio de identidad (Katzenstein, Keohane \& Krasner, 1999). Wendt, por ejemplo, define la identidad como el entendimiento sobre los roles específicos y expectativas acerca de sí mismo y del otro. Las identidades forman los intereses y estos se crean en los procesos de interacción, donde también surge la identidad (Wendt, 1999), demostrando que tienen una relación de mutua constitución (Finnemore, 1996). De esta manera, los intereses nacionales se constituyen a partir de la identidad del país y la construcción de la identidad respecto del otro (Weldes, 1996).

La identidad es también un factor fundamental en el análisis pospositivista, aunque su forma de tratarla es diferente, siendo que la identidad es un factor que se utiliza para entender la constitución de límites adentro/afuera y de relaciones de poder (Campbell, 1990). Este aspecto diferencia estas vertientes críticas del mismo constructivismo mainstream, que de alguna manera analiza los procesos de construcción de identidad de forma acrítica, sin reconocer las relaciones de poder que se dan detrás de esos procesos. Análisis como los de Campbell (1990), Doty (1993) y Weldes (1996) resaltan la importancia de los procesos constitutivos de identidad y cómo esto repercute en relaciones de poder a partir de la construcción de imaginarios sobre el otro. 
Teniendo eso en cuenta, el aporte de estos enfoques para el caso en cuestión es que permiten entender mejor cómo la indigeneidad es utilizada en el discurso como una forma de cuestionar las relaciones de poder de una manera más profunda que la que se analiza tradicionalmente en las relaciones internacionales y es, a su vez, una forma de ejercer el poder por medio del discurso, pero al mismo tiempo, al ser un proceso de construcción de identidad desde arriba, muestra cómo se ejercen las relaciones de poder.

A pesar de esto, el problema de muchos de los enfoques pospositivistas, como posestructuralismo, es que se quedan cortos para analizar la política exterior desde otras cosmovisiones. Al partir y aceptar las categorías propias de la disciplina, no logran explicar, o por lo menos no se preocupan por explicar, la constitución de la identidad a partir de relaciones más allá de las humanas, y la politización de los otros mundos, propios de las cosmovisiones indígenas, como factores constitutivos de identidad y de política exterior como sucede con el caso boliviano.

Es evidente que la indigeneidad se utiliza como factor de identidad y como interés nacional, y, de esta manera, la utilización de esta en la política exterior no solo se da respecto a los otros países sino respecto a los otros gobiernos antecesores y también con el objetivo de construir nación, siendo entonces que uno de los públicos objetivo de este discurso es la misma población boliviana. Pero la indigeneidad, en cierta medida, es también una forma que plantea el gobierno de autoencontrarse, donde la recuperación de la memoria y del legado ancestral indígena es un interés nacional; es un elemento necesario, porque se refiere a la identidad política y cultural que el país quiere mostrar, y también es un interés variable, que se concreta recién con este gobierno, ${ }^{9}$ lo que muestra la importancia que tienen los entornos domésticos e internacionales en la construcción de la identidad y el interés nacional (Jepperson, Wendt \& Katzenstein, 1999).

9 Sobre la clasificación de los intereses ver Morgenthau (1990) y Neuchterlein (1976). 
La indigeneidad puede ser también, en términos de Neuchterlein (1976), un interés ideológico, en la medida en que busca rescatar valores que la gente comparte o en los que cree. Ahora bien, la clasificación de intereses de Neuthterlein se aplica muy bien para el caso en cuestión, porque la indigeneidad se usa tanto como reivindicación, como con fines pragmáticos, y se plasma en la satisfacción de necesidades que se traducen en mejores condiciones económicas o de seguridad. Aquí es donde puede verse que, si bien la indigeneidad puede tener un fuerte contenido ideológico, también es utilizada estratégica y pragmáticamente por el Gobierno para abrirse espacios en escenarios multilaterales, y asumir un liderazgo regional. Dentro de su pragmatismo, el Gobierno es consciente de que, en muchos escenarios políticos internacionales, el aspecto indígena es aún visto como algo pintoresco y exótico, que no hace daño a nadie, es algo atractivo y no amenazante, su alcance e impacto se da más a nivel social (tiene eco en diferentes poblaciones del mundo) que a nivel intergubernamental. La indigeneidad le ha permitido a Bolivia ampliar su agenda y diversificar su política exterior. Por ejemplo, ha tomado la iniciativa y ha sido anfitrión de la conferencia Mundial de los Pueblos sobre Cambio Climático en Cochabamba el 2010 (Birns \& Sánchez, 2011).

Es necesario afirmar, sin embargo, que basar un interés nacional en aspectos de identidad, como la indigeneidad es complejo y muy problemático, pues siempre será una imposición para quienes no comparten dicha identidad, y a la larga puede ser un factor de conflicto interno, o por lo menos de serias tensiones regionales a nivel subnacional. En el ámbito doméstico, las políticas de desarrollo del Gobierno chocan con los principios indigenistas del buen vivir y atentan contra las formas de vida de distintos pueblos en el país. Como se dijo antes, en Bolivia algunos grupos étnicos son históricamente más fuertes que otros, o consideran que los otros son inferiores. Así las cosas, si bien se habla de una "diplomacia de los pueblos" y se rescatan varios conocimientos y valores ancestrales de muchos pueblos, el presidente no es "indígena" sino aymara, y toda su política exterior es aymarocentrista con aspiración nacionalista (Alvizuri, 2009). En otras palabras, instrumentaliza su autoidentificación como aymara 
para la construcción de un nacionalismo, que representa a un pueblo mayoritario pero que lo enfrenta a las minorías étnicas a las cuales dice representar. La política exterior se convierte en un instrumento utilizado por el Gobierno en la que la indigeneidad aparece como un factor de cohesión social que muestra una imagen al mundo de un país mayoritariamente indígena, cuando en realidad no se reduce a eso.

\section{Conclusiones}

En términos generales, la política exterior de Bolivia con el gobierno de Evo Morales puede ser calificada de revisionista. Esto, porque hay una marcada ruptura con alianzas y relaciones que anteriores gobiernos habían privilegiado y porque Bolivia se inserta en el grupo de países de la región que optan por un giro ideológico hacia la izquierda, lo que implica una nueva posición respecto a los tratados de libre comercio y a las relaciones diplomáticas en general. También es una política revisionista en la medida en que el país se ha puesto en la tarea de revisar los compromisos previamente asumidos que, de alguna manera, perpetúan una relación de colonialidad o que atentan con el principio de soberanía.

Finalmente, puede decirse que la política exterior boliviana es subversiva en la medida en que innova, al utilizar la indigeneidad como interés nacional y sobre todo por lo que esto implica cuestionar la cultura, civilización y modelo económico occidental, al proponer retomar la sabiduría ancestral de los pueblos para hacer frente a la crisis en la que la humanidad se encuentra. Esto, sin duda es muy provocativo y contestatario, pero es muy poco probable que vaya a alterar en algo el orden internacional. A su vez, la política exterior boliviana es marcadamente pragmática en el sentido en que existe un doble discurso, sobre todo en lo económico y ambiental. En la política exterior boliviana existen profundas contradicciones, de las cuales la más evidente es la posición del país frente a la explotación y exportación de recursos y el discurso de protección del medio ambiente con base en la recuperación de los conocimientos ancestrales de los pueblos. Pero en esto Bolivia no es la excepción. Las contradicciones en política exterior son muy frecuentes en muchos países. 
La utilización de la indigeneidad en la política exterior, a partir de procesos de construcción de identidad y de buscar defender un interés nacional es un arma de doble filo. Por un lado, le ha permitido a Evo Morales iniciar una búsqueda de justicia y reivindicación que pasa por implementar cambios domésticos e internacionales que tienen que ver con la recuperación de la memoria y el reconocimiento del legado indígena, que a su vez han posibilitado acercamientos con otros países y otros pueblos; pero, por otro, al ser tan directamente dirigida por el Gobierno, se convierte en un proceso concebido e implementado desde arriba, lo que repercute en la marginación de grupos minoritarios y acalla voces disidentes.

\section{Referencias}

Alden, C., \& Aran, A. (2012). Foreign Policy Analysis. New Approaches. Nueva York: Routledge.

Alvizuri, V. (2009). Mecanismos de cristalización aymara en Bolivia. En V. Robin \& Salazar, C. (Eds.), El regreso de lo indígena. Retos, problemas y perspectivas. Actes y memoires 26 (pp. 135-156). Lima: Instituto Francés de Estudios Andinos.

Birns, L., \& Sánchez, A. (2011). From Obscurity to Center Stage: The Architectonics of Bolivia's Foreign Policy. En G. L. Gardini \& P. Lambert (Eds.), Latin American Foreign Policies, Between Ideology and Pragmatism (pp. 103-118). Nueva York: Palgrave Macmillan.

Burchill, S. (2005). The National Interest in International Relations Theory. New York: Palgrave Macmillan.

Campbell, D. (1990). Global Inscription: How Foreign Policy Constitutes the United States, Alternatives, 15, 263-286.

Choquehuanca, D. (2011). Entrevista a David Choquehuanca. Recuperado de http:/ /www.youtube.com/watch?v=epHOudAdV4Y

Choquehuanca, D. (2012). Los pilares de la nueva politica exterior boliviana. Recuperado de http://www.casamerica.es/politica/los-pilares-de-lanueva-politica-exterior-boliviana

De la Cadena, M. (2010). Indigenous cosmopolitics in the Andes: Conceptual Reflections beyond 'Politics'. Cultural Antbropology, 25(2), 334-370. De la Cadena, M., \& Stran, O. (Eds.) (2007). Indigenous experience today. Oxford: Berg. 
Doolittle, A. (2010). The Politics of Indigeneity: Indigenous Strategies for Inclusion in Climate Change Negotiations. Conservation and Society, 8(4), 286-291.

Dunne, Tim; Hadfield, Amelia \& Smith, Steve (eds.). (2011). Foreign Policy: Theories, Actors, Cases. Oxford: Oxford University Press.

Escobari, J. (1999). Historia Diplomática de Bolivia. La Paz: Editorial Urquizo. Estado Plurinacional de Bolivia (2009). Nueva Constitución Política del Estado Boliviano. Recuperado de http://www.comunicacion.gob. bo/?q=20130725/nueva-constitucion-politica-del-estado-boliviano Estermann, J. (2012). Crisis civilizatoria y vivir bien. Una crítica filosófica del modelo capitalista desde el allin kawsay/suma qamaña andino, Polis, 11(33), 1-18.

Finnemore, M. (1996). National Interests in International Society, Ithaca: Cornell University Press.

Foro Permanente de las Naciones Unidas para las Cuestiones Indígenas. (Abril-mayo de 2009). Misión a Bolivia. Informe y recomendaciones. Recuperado de http://www.un.org/esa/socdev/unpfii/documents/ UNPFII_Mission_Report_Bolivia_ES.pdf Fecha de acceso 18 de marzo de 2014

Franke, M. F. 2009. The Political Stakes of Indigenous Diplomacies. Questions of Difference. In M. Beier (Ed.). Indigenous Diplomacies. New York: Palgrave Macmillan.

González Pazos, J. (2007). Bolivia. La construcción de un país indígena. Barcelona: Icaria Editorial S.A.

Huacanuni, F. (2012). Suma Qamaña o vivir bien. Recuperado de http:/ /www. youtube.com $/$ watch?v $=90$ ZHJMTcfOE

Jepperson, R., Wend, A., \& Katzenstein, P. (1999). Norms, Identity, and Culture in National Security. In P. J. Katzenstein, (Comp.), The Culture of National Security: Norms and Identity in World Politics. (pp. 33-75). New York: Columbia University Press.

Kaarbo, J. (2003). Foreign Policy Analysis in the Twenty-First Century: Back to Comparison, Forward to Identity and Ideas. Foreign Policy Analysis in 20/20: A Symposium. International Studies Review 5, 155-202.

Katzenstein, P. J. (Comp.) (1996). The Culture of National Security: Norms and Identity in World Politics. New York: Columbia University Press.

Katzenstein, P. J., Keohane, R. O., \& Krasner, S. (Eds.) (1999). Exploration and Contestation in the Study of World Politics. Cambridge, MA: MIT Press. 
Kohl, B. (2010). Bolivia under Morales: A Work in Progress, Latin American Perspective, 37(107), 107-122.

Laurent, V. (2005). Comunidades indígenas, espacios politicos y movilización electoral en Colombia, 1990-1998. Motivaciones Campos de Acción e impactos. Bogotá: Instituto Colombiano de Antropología e Historia.

Ministerio de Relaciones Exteriores de Bolivia: Programa "Un futuro sembrado hace miles de años", año internacional de la quinua 2013. Recuperado de http://www.quinuainternacional.org.bo/

Maira, L. (2007). Dilemas internos y espacios internacionales en el gobierno de Evo Morales, Nueva Sociedad, 209, 65-81

Merlan, F. (2009). Indigeneity, Current Anthropology, 50(3), 303-333.

Moldiz, H. (2007). Bolivia: crisis estatal y proceso de transformación. En B. Stolowicz (Coord.), Gobiernos de izquierda en América Latina. Un balance politico. (pp. 155-196). Bogotá: Ediciones Aurora.

Morgenthau, H. (1990). "Otro gran debate: el interés nacional de los Estados Unidos”. En H. Morgenthau. Escritos sobre politica internacional. (pp. 99-109). Madrid: Tecnos.

Neuchterlein, D. (1976). National Interests in Foreign Policy: A Conceptual Framework for Analysis and Decision-Making. British Journal of International Studies, 2(3), 246-266.

Patxi, F. (2009). Bolivia: Entrevista con el pedagogo boliviano Félix Patzi Paco Revista Gloobal Hoy. Recuperado de http://www.gloobal.net/ iepala / gloobal $/$ fichas / ficha.php?entidad=Textos\&id=10676

Quispe, F. (15 de junio de 2014). Yo, Felipe Quispe, soy culpable del ascenso de Evo. Pagina Siete. Recuperado de http:/ /www.paginasiete.bo/nacio$\mathrm{nal} / 2014 / 6 / 15 /$ yo-felipe-quispe-culpable-ascenso-evo-24244.html

Rochlin, J. (2007). Latin America's Left Turn and the New Strategic Landscape: The Case of Bolivia, Third World Quarterly, 28(7), 1327-1342.

Shaw, K. (2002). Indigeneity and the International. Millennium - Journal of International Studies, 31(1), 55-81.

Smith, S., Hadfield, A., \& Dunne, T. (2011). Foreign Policy. Theories, actors, cases. Oxford: Oxford University Press.

Trigger, D., \& Dalley, C. (2010). Negotiating Indigeneity: Culture, Identity, and Politics, Reviews in Anthropology, 39(1), 46-65.

Vecchione, Marcela. 2009. Between the leader of Virtu and the Good Savage. Indigenous struggles and Life projects in the Amazon Basin. En M. Beier ( Ed.). Indigenous Diplomacies. New York: Palgrave MAcmillan. 
Viceministerio de la Coca y el Desarrollo Integral. (2014). Misión y Visión. Recuperado de http://www.vcdi.gob.bo/

Viceministerio de Descolonización de Bolivia. (2014). Recuperado de http://www.descolonizacion.gob.bo/

Weldes, J. (1996). Constructing National Interests. European Journal of International Relations, 2(3), 275-318.

Wendt, A. (1999). Social Theory of International Politics. Cambridge: Cambridge University Press. 